\title{
The effect of context on visual representational momentum
}

\author{
TIMOTHY L. HUBBARD \\ Eastern Oregon State College, La Grande, Oregon
}

\begin{abstract}
Effects of background context on representational momentum were examined in six experiments. In each experiment, three orientations of a target rectangle undergoing implied rotation (i.e., the inducing stimuli) were presented, and subjects judged whether the orientation of a fourth rectangle (i.e., the probe) was the same as or different from that of the third inducing stimulus. Target rectangles were enclosed within a larger square frame context during induction (i.e., presentation of the inducing stimuli), judgment (i.e., presentation of the probe), or both induction and judgment. If context during induction moved in the same direction as the inducing stimuli or if context during judgment was rotated slightly forward from the orientation of the final inducing stimulus, representational momentum was increased. If context during induction moved in the direction opposite to the inducing stimuli or if context during judgment was rotated slightly backward from the orientation of the final inducing stimulus, representational momentum was decreased or reversed. If context was present during both induction and judgment, direction of representational momentum was biased toward the context at judgment. Implications of context for accounts of representational momentum are discussed, and a tentative model is proposed.
\end{abstract}

Subjects' memory for the final orientation of an object undergoing implied rotation (Freyd \& Finke, 1984) or the final location of a linearly moving object that vanishes without warning (Hubbard, 1990; Hubbard \& Bharucha, 1988 ) is systematically shifted in the direction of object motion. The magnitude and direction of this memory shift are influenced by a number of factors: implied velocity (Freyd \& Finke, 1985; Hubbard \& Bharucha, 1988) and acceleration (Finke, Freyd, \& Shyi, 1986) of the object, direction of implied motion (Hubbard, 1990), constancy of object shape (Kelly \& Freyd, 1987), the amount of time between disappearance of the object and probing of remembered location (Freyd \& Johnson, 1987), coherence of inducing sequence (Freyd \& Finke, 1984), similarity of the final stimulus to a prototypical member of a category (Kelly \& Freyd, 1987) or a schematically proper ending (Hubbard, 1992a), and whether implied motion of the object is embedded in an event hierarchy (Verfaillie \& d'Ydewalle, 1991). This memory shift has been referred to as representational momentum in studies involving remembered orientation of objects undergoing implied rotation (Freyd \& Finke, 1984; Kelly \& Freyd, 1987) or remembered pitch (Freyd, Kelly, \& DeKay, 1990; Hubbard, 1992a, 1992b) and as displacement in studies involving remembered location of objects undergoing

This research was supported by general research funds from Eastern Oregon State College. Portions of this research were presented at the 32nd Annual Meeting of the Psychonomic Society, November 1991. The author thanks James Chumbley, Gery d'Ydewalle, Pierre Jolicoeur, and Karl Verfaillie for helpful comments on an earlier version of the manuscript and Deana Lilly for assistance in data collection. Correspondence should be addressed to T. Hubbard, Department of Psychology, Eastern Oregon State College, La Grande, Oregon 97850 (e-mail: thubbard@oregon.uoregon.edu). apparent linear motion (Hubbard, 1990; Hubbard \& Bharucha, 1988).

In the typical paradigm for assessing visual representational momentum, subjects see three brief discrete sequential presentations of a rectangle. These rectangles are referred to as the "inducing stimuli," and each rectangle is presented at a slightly different orientation. Subjects are then shown a fourth "probe" rectangle and must judge whether the probe is at the same orientation as the third inducing stimulus or a different orientation. The probe may be at the same orientation as the third inducing stimulus, or it may be at an orientation slightly beyond the orientation of the third inducing stimulus (i.e., rotated forward from the orientation of the third inducing stimulus in the direction of implied rectangle rotation) or behind the orientation of the third inducing stimulus (i.e., rotated backward from the orientation of the third inducing stimulus in the direction opposite to the implied rectangle rotation). Typically, when the sequence of inducing stimuli is such that motion in a consistent direction is implied, subjects are more likely to respond erroneously same to probes oriented slightly beyond the orientation of the third inducing stimulus than to probes oriented slightly behind the orientation of the third inducing stimulus.

The magnitude and direction of representational momentum can be influenced by the context in which the inducing stimuli are seen; specifically, if a context of periodic changes in the direction of movement is established, subjects' judgments will be displaced in ways consistent with that context. For example, Bharucha and Hubbard (1992) presented subjects with a moving circular target enclosed within a large square box. The target bounced off the inner walls of the box between one and five times before both the box and target simultaneously vanished. 
When the target vanished just prior to or at the moment of collision with a wall of the box, subjects' judgments of the vanishing points were displaced in a pattern that suggested they had extrapolated the probable future path of the target and had already "bounced." Verfaillie and d'Ydewalle (1991) showed similar effects of expectation of future target behavior on representational momentum by presenting subjects with inducing sequences that implied either periodic or aperiodic motion. Representational momentum decreased to near zero when the target was near the boundary of a periodic motion and about to reverse direction (hence the derivational velocity of the final inducing stimulus was zero), but a standard forward representational momentum shift was observed when the target was undergoing an aperiodic motion or was far from the boundary of a periodic sequence and continuing in the same direction (hence derivational velocity of the final inducing stimulus was greater than zero). In both the Bharucha and Hubbard and the Verfaillie and d'Ydewalle experiments, the "inducing context" of a target either repeatedly bouncing or undergoing periodic motion channeled subjects' representational momentum along pathways consistent with a future anticipated target behavior. In these studies, context was thus very broadly defined and "top down" in its influence (see also Hubbard, 1992d).

A more limited type of context not explicitly addressed in previous studies involves physical elements that could function as a physical "background" to the target "figure" in the stimulus display. For example, studies using the Freyd and Finke rotating rectangle paradigm have typically not included any other elements in the display, and with only a few exceptions (e.g., Finke \& Freyd, 1985; Finke et al., 1986), investigation of visual representational momentum has focused on memory displacements of single isolated elements in a scene. If, however, an individual element in the foreground of a visual scene (such as a rectangle or a circular target) is capable of exhibiting representational momentum, then it is possible that additional elements in the background, if not the entire background context, might contribute to or otherwise modify the representational momentum of the target. It is also possible that any effects of background display context on representational momentum may depend in part on the timing of when the context is present; for example, context may have different effects depending on whether it is presented concurrently with the inducing stimuli or concurrently with the judgment of the probe.

\section{EXPERIMENT 1}

In this experiment, subjects were presented with the standard Freyd and Finke rotating rectangle. The rectangle was enclosed within a larger square frame that was visible throughout the presentation of both the inducing stimuli and the probe. The frame was at one of four orientations on each trial: upright, rotated backward (i.e., in the direction opposite to target rotation) by $4^{\circ}$ from the orientation of the third inducing stimulus, at the same orientation as the third inducing stimulus, or rotated forward (i.e., in the same direction as target rotation) by $4^{\circ}$ from the orientation of the third inducing stimulus. If the context (i.e., the orientation of the surrounding frame) had no influence on representational momentum of an object within that context, then we would not expect any difference in the representational momentum pattern as a function of whether the frame was present. If, however, the context did influence representational momentum (perhaps by providing specific orientation information), then we would expect slightly stronger representational momentum when the frame was oriented slightly beyond the orientation of the third inducing stimulus than when it was oriented slightly behind the orientation of the third inducing stimulus. Alternatively, the frame might diminish representational momentum by providing a generic landmark for subjects to use.

\section{Method}

Subjects. The subjects were 12 undergraduates from Eastern Oregon State College who participated in return for extra credit in an introductory psychology course.

Apparatus. The stimuli were displayed upon and data stored in an Apple Macintosh IIcx microcomputer equipped with an Apple RGB color monitor. The subjects could adjust the viewing distance in order to achieve maximum comfort and confidence in their responses.

Stimuli. The target was a black outline rectangle on a white background. The target was centered in the approximate middle of the screen; the long axis of the target was 90 pixels (approximately $3.75^{\circ}$ ), and the short axis was 30 pixels (approximately $1.25^{\circ}$ ) in length. On each trial, the target was presented in three "inducing" orientations and a fourth "probe" orientation. Consistent with the procedures of Freyd and Finke (1984) and Kelly and Freyd (1987), the first inducing rectangle was presented with its long axis oriented vertically, the second inducing rectangle was oriented $17^{\circ}$ from the orientation of the first inducing rectangle, and the third inducing rectangle was oriented $34^{\circ}$ from the first inducing rectangle such that motion in either a consistent clockwise or consistent counterclockwise direction was implied. Each inducing rectangle was presented for $250 \mathrm{msec}$, and the interstimulus interval (ISI) between presentations of the stimuli was also $250 \mathrm{msec}$. The probe remained visible until the subject responded, and it appeared at one of five orientations relative to the orientation of the third inducing rectangle: $-4^{\circ},-2^{\circ}, 0^{\circ},+2^{\circ}$, or $+4^{\circ}$. The inducing rectangles and the probe were surrounded by a square frame such that the center of the frame was the same as the centers of the inducing rectangles and the probe. The frame was 200 pixels (approximately $8.33^{\circ}$ ) along each side. The frame was at one of four orientations relative to the orientation of the third inducing rectangle: upright (i.e., with lines oriented horizontally and vertically), $-4^{\circ}, 0^{\circ}$, or $+4^{\circ}$. For both probe orientations and frame orientations, negatively signed orientations indicate orientations behind the true-same orientation (i.e., behind the rotating rectangle and through which implied motion would have already passed), whereas positively signed orientations indicate orientations beyond the true-same orientation (i.e., beyond the rotating rectangle and through which implied motion would not have yet passed). For example, a probe at $-4^{\circ}$ would be clockwise from the orientation of the final inducing stimulus in a counterclockwise trial, but counterclockwise from the orientation of the final inducing stimulus in a clockwise trial. A probe orientation or frame orientation of $0^{\circ}$ indicates a probe or frame at the true-same orientation (i.e., the same orientation as the third inducing rectangle). The frame appeared when the first inducing rectangle 


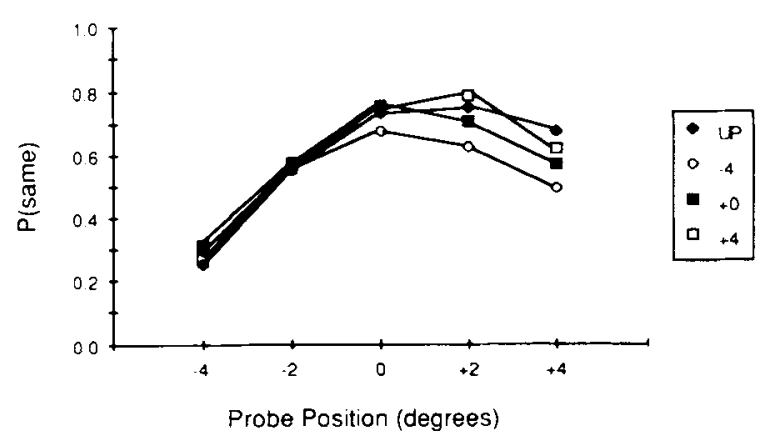

Figure 1. Probability of a same response as a function of probe orientation in Experiment 1.

appeared and remained visible for the duration of that trial. Each subject received 400 trials ( 2 directions $\times 5$ probe orientations $\times$ 4 frame orientations $\times 10$ replications) in a different random order.

Procedure. The subjects were first given a practice session consisting of 12 trials that were randomly selected from the experimental trials. The subjects initiated each trial by pressing a designated key, and the inducing stimuli and surrounding frame immediately appeared. After the probe appeared, the subjects judged whether its orientation was the same as or different from the orientation of the third inducing stimulus. They were instructed to be as accurate as possible and were allowed as much time as they required. The subjects were told it was possible that there would be more same than different, equal numbers of same and different, or fewer same than different orientations. They pushed either a key marked $S$ or a key marked $D$ to indicate their response.

\section{Results and Discussion}

The choice probabilities are displayed in Figure 1. Were subjects responding accurately, there would be $0 \%$ same responses on negatively and positively signed probe orientations and $100 \%$ same responses on $0^{\circ}$ unsigned orientations. Estimates of the degree of memory shift were determined by calculating the weighted mean (i.e., the sum of the products of the proportion of same responses and the distance of the probe from true-same, in degrees, divided by the sum of the proportions of test responses), and the shift estimates are listed in Table 1.

The shift estimates were analyzed in a 2 (direction) $x$ 4 (frame orientation) repeated measures analysis of variance (ANOVA), and the alpha level required for significance was set at .05 . Frame orientation significantly influenced the degree of shift $\left[F(3,33)=5.97, M S_{\mathrm{e}}=\right.$ .127]; post hoc Newman-Keuls tests revealed that the upright condition $(x=.69)$ led to more forward shift than did the $-4^{\circ}(x=.31)$, true-same $(x=.40)$, or $+4^{\circ}$ $(x=.59)$ conditions, and the $+4^{\circ}$ condition led to more forward shift than did the $-4^{\circ}$ condition. As can be seen in Figure 1, when the frame is either upright or $4^{\circ}$ beyond true-same, forward shift size is relatively larger, but when the frame is at true-same or rotated backward from true-same, shift size is relatively smaller (although still positive). No other factors were significant (all $F \mathrm{~s}<$ 2.0 , all $p s>.14$ ).

The data support the notion that the context surrounding an object can influence the pattern of representational momentum for that object. When the frame was rotated backward, representational momentum was decreased, but when the frame was rotated forward, representational momentum was increased. Thus, when the context is centered around the orientation of the final inducing stimulus and is such as to suggest a particular orientation, subjects' representational momentum is more likely to be shifted in the orientation of the context. The larger shift with the upright frame is more surprising, but might be interpreted as suggesting that the distance between the context and the target influences representational momentum. This notion is bolstered by considering the edges of the monitor screen as an "upright condition," albeit an upright condition in which the context is more distant from the stimuli. The relatively large shift in the upright condition of Experiment 1 might then result from having the upright context closer to the target. Such a notion would predict that as distance between the frame and the target de-

Table 1

Estimated Representational Momentum* as a Function of Frame Orientation

\begin{tabular}{ccccccc}
\hline & \multicolumn{5}{c}{ Estimated Representational Momentum as a Function of Frame Orientation } \\
\cline { 2 - 6 } Experiment & $-4^{\circ}$ & $-2^{\circ}$ & $0^{\circ}$ & $+2^{\circ}$ & $+4^{\circ}$ & Upright \\
\hline 1 & $.31^{\circ}$ & & $.40^{\circ}$ & & $.59^{\circ}$ & $.69^{\circ}$ \\
2 & $.19^{\circ}$ & & $.12^{\circ}$ & & $.20^{\circ}$ & $.10^{\circ}$ \\
3 & $-.54^{\circ}$ & $-.10^{\circ}$ & $.49^{\circ}$ & $.90^{\circ}$ & $1.26^{\circ}$ & \\
$4 \ddagger$ & $-.55^{\circ}$ & $-.47^{\circ}$ & $.10^{\circ}$ & $.69^{\circ}$ & $1.30^{\circ}$ & \\
6 & $-.44^{\circ}$ & $-.31^{\circ}$ & $.01^{\circ}$ & $.24^{\circ}$ & $.48^{\circ}$ & \\
\hline
\end{tabular}

*The sign of the representational momentum estimates indicates the direction of shift. Negative values indicate shifts in the direction opposite to the direction of target motion (i.e., behind the true-same orientation), and positive values indicate shifts in the direction of target motion (i.e., beyond the true-same orientation). †The sign of the frame orientation indicates the relationship between the frame orientation and the orientation of the final inducing stimulus. Negative values indicate orientations behind the orientation of the final inducing stimulus (i.e., rotated backward in the direction opposite to target motion), and positive values indicate orientations beyond the orientation of the final inducing stimulus (i.e., rotated forward in the direction of target motion). $\mathrm{A}^{\circ}$ value indicates an orientation identical to the orientation of the final inducing stimulus. In the upright condition, the lines of the frame were oriented horizontally and vertically. †Judgment condition. 
creased, the influence of the context would increase. $\mathrm{Al}$ ternatively, the larger representational momentum in the upright condition might be accounted for if we consider a rotated frame to have two effects: (1) an overall diminution of momentum that is independent of frame orientation and (2) an effect of frame orientation that influences momentum in the direction of the orientation. The former effect might occur because of interference of the frame with a standard "upright" reference, and this additional noise would make the response distribution more symmetric because of unbiased random perceptual noise. The second effect would be the predicted effect of context orientation.

\section{EXPERIMENT 2}

The previous experiment established that the context can influence the pattern of representational momentum, but because the frame was visible during the presentation of both the inducing rectangles and the probe, it was not clear whether the effects of context were operative during induction or judgment, or perhaps during both induction and judgment. When Bharucha and Hubbard (1992) removed the motion of the target from their displays and briefly presented stationary targets at locations corresponding to the precollision, collision, and postcollision vanishing points, the displacement patterns were very different than when subjects saw motion up to the vanishing points, a result suggesting that at least some of the experience during induction (i.e., the experience of motion) does play a role in representational momentum. In this experiment, context (i.e., the frame) was visible during the presentation of the inducing stimuli but not during the presentation of the probe. If context was operative only during judgment, then no effect of frame orientation should be observed. If, however, context did influence representational momentum during induction, then effects of frame orientation should be observed.

\section{Method}

Subjects. The subjects were 12 undergraduates drawn from the same pool as in Experiment 1, and none of the subjects had participated in Experiment 1.

Apparatus. The apparatus was the same as in Experiment 1.

Stimuli. The stimuli were the same as in Experiment 1, with the following exception: the frame appeared when the first inducing rectangle appeared and vanished when the third inducing rectangle disappeared. Each subject received 400 trials $(2$ directions $\times 5$ probe orientations $\times 4$ frame orientations $\times 10$ replications) in a different random order.

Procedure. The procedure was the same as in Experiment 1, with the following exception: the frame vanished when the third inducing rectangle vanished, and the frame was not visible during the presentation of the probe.

\section{Results and Discussion}

The choice probabilities are displayed in Figure 2. Estimates of memory shift were calculated as in Experiment 1 and are listed in Table 1. Shift estimates were analyzed as in Experiment 1. Neither direction, frame

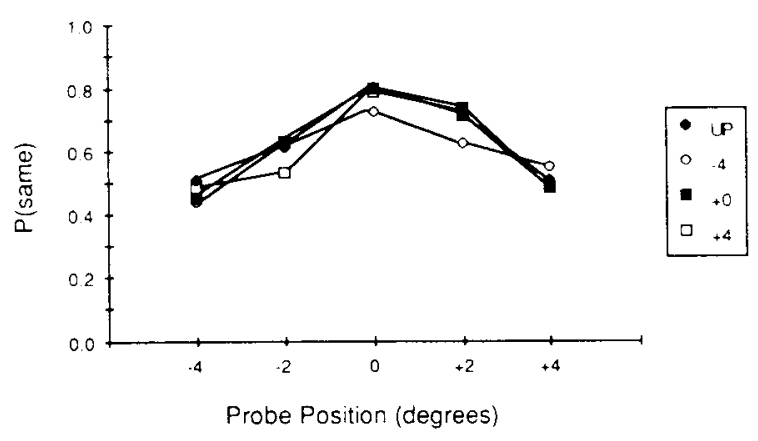

Figure 2. Probability of a same response as a function of probe orientation in Experiment 2.

orientation, nor the direction $\times$ frame orientation interaction attained significance (all $F \mathrm{~s}<.64$, all $p \mathrm{~s}>.59$ ). The failure of frame orientation to significantly influence the memory shift suggests that the frame must be present during judgment to have an effect and that any effects during induction are relatively weak.

Overall, the data are consistent with the notion that the context at judgment can influence the pattern of representational momentum. In Experiment 1, when the context was present during both induction and judgment and was such as to suggest a particular orientation, the subjects' representational momentum was more likely to be shifted toward the frame orientation. In Experiment 2, when the context was present only during induction, significant differences in representational momentum were not obtained. One possible explanation for the effects is that the frame during judgment may have functioned as a landmark of some sort. In Experiment 1, when the frame was rotated forward, remembered probe orientation would have been relatively close to the frame orientation, and so remembered probe orientation might have been more likely to be assimilated to the frame orientation. When the frame was rotated backward, however, remembered probe orientation would have been relatively far from the frame orientation, and so remembered probe orientation might have been less likely to be assimilated to the frame orientation. The frame may, nonetheless, have functioned as a landmark or an anchor even if it was rotated backward (i.e., orientation was a little beyond this point). In Experiment 2, however, the frame was not present during judgment, so the frame was not able to function as a landmark or anchor during judgment, and so it did not influence the subjects' responses as it did in Experiment 1.

In Experiments 1 and 2, the context was stationary, but what would happen if the context itself were in motion? Such often occurs in the "real world"; for example, an observer in motion watching other objects experiences parallax in which objects appear to move with a velocity that is in part a function of their distance from the observer. Similarly, a stationary observer watching two objects of similar velocities but different distances would also perceive parallax. If the context was such as to sug- 
gest motion in the same direction as the motion of the target, and the effects of motion in the target combined with the effects of motion in the context, it is possible that representational momentum could be even greater than if the target moved within a stationary context. Alternatively, forward representational momentum derived from motion of the context might nullify forward representational momentum derived from motion of the target.

\section{EXPERIMENT 3}

In the previous experiments, the context present during presentation of the inducing stimuli was stationary; the frame was either upright or at $-4^{\circ}, 0^{\circ}$, or $+4^{\circ}$ relative to the orientation of the third inducing stimulus. This experiment examined. whether motion of the frame itself can influence the representational momentum of the target. Faust (1990) presented a moving rectangular context that surrounded a smaller stationary target and found that memory for the location of the stationary target was shifted in the direction opposite to motion of the rectangular context. Faust's results are consistent with examples of induced motion, but because he was looking at shifts in the memory for the target's position, rather than at judgments of whether the target moved, his results are also consistent with representational momentum (i.e., the "induced motion" of the target led to memory for the target's position being displaced in the direction of perceived motion). Faust's results suggest that motion in a surrounding context can influence the representational momentum of a stationary target to the extent that the motion of the context evokes a perception of motion of the target, but his data do not shed light on how motion in a surrounding context can influence the representational momentum of a target that is already in motion.

In this experiment, a new frame orientation was presented with each presentation of an inducing stimulus or a probe. During presentation of each of the inducing stimuli, a frame was presented at the same orientation as the inducing rectangle. During presentation of the probe, the frame was at one of five orientations on each trial: rotated backward by $4^{\circ}$ or $2^{\circ}$ from the orientation of the third inducing stimulus, at the true-same orientation as the third inducing stimulus, or rotated forward by $2^{\circ}$ or $4^{\circ}$ from the orientation of the third inducing stimulus. If motion of the context during induction had no influence on representational momentum of a target stimulus within that context, then we would not expect any difference in the pattern of representational momentum from that found in Experiment 1. If, however, motion of the context during induction did influence or combine with motion in a target, then we would expect a different pattern of representational momentum from that found in Experiment 1.

\section{Method}

Subjects. The subjects were 13 undergraduates drawn from the same pool as in Experiment 1, and none of the subjects had participated in previous experiments.
Apparatus. The apparatus was the same as in Experiment 1. Stimuli. The stimuli were the same as in Experiment 1, with the following exception: the frames appeared and disappeared with each appearance and disappearance of the inducing and probe stimuli. During the presentation of each inducing rectangle, the frame was presented at the same orientation as the inducing rectangle. During the presentation of the probe, the frame was at $-4^{\circ},-2^{\circ}, 0^{\circ},+2^{\circ}$, or $+4^{\circ}$ from the orientation of the third inducing rectangle. Each subject received 500 trials ( 2 directions $\times 5$ probe orientations $\times$ 5 frame orientations $\times 10$ replications) in a different random order.

Procedure. The procedure was the same as in Experiment 1, with the following exception: the frame was visible only during the presentation of each inducing stimulus and the probe and was not visible during the ISIs between presentations.

\section{Results and Discussion}

Choice probabilities are displayed in Figure 3. Shift estimates were calculated as in Experiment 1 and are listed in Table 1 . The shift estimates were analyzed in a 2 (direction) $\times 5$ (frame orientation) repeated measures ANOVA, and the alpha level required for significance was set at .05. Frame orientation significantly influenced the magnitude of representational momentum $[F(4,48)=14.31$, $\left.M S_{\mathrm{e}}=.97\right]$, and post hoc Newman-Keuls tests $(p<.05)$ revealed that all pairwise comparisons except for $\left(-4^{\circ}\right.$, $\left.-2^{\circ}\right)$ and $\left(0^{\circ},+2^{\circ}\right)$ were significant. As shown in Figure 3 , when the frame orientation is rotated backward from the true-same, memory shift is backward, and when the frame orientation is rotated forward from the truesame, memory shift is forward; furthermore, the magnitude of representational momentum was influenced by how far the orientation of the frame was from true-same: larger distances led to larger magnitudes of representational momentum. Direction was also significant $\left[F(1,12)=7.22, M S_{e}=.15\right]$, with clockwise motion $(x=.49)$ resulting in larger overall shifts than counterclockwise motion $(x=.32)$. The direction $\times$ frame orientation interaction was not significant $[F(4,48)=.69$, $M S_{\mathrm{e}}=.09$ ].

The data suggest that if the context is moving in the same direction as the target stimulus, overall representational momentum may be enhanced. Additionally, the differences between representational momentum patterns for frame orientations rotated forward and for frame orien-

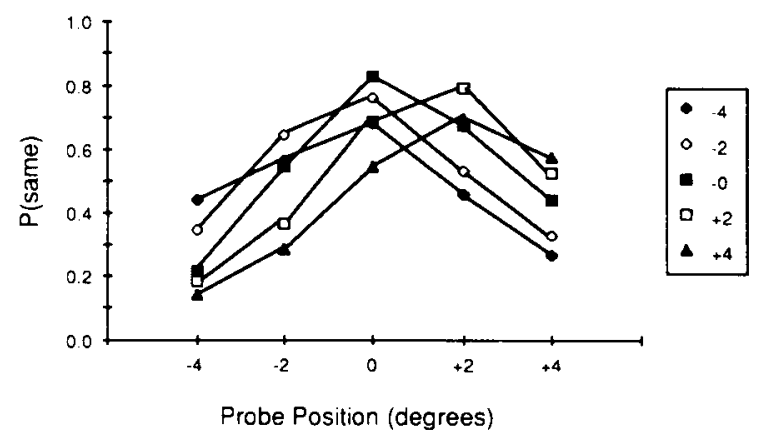

Figure 3. Probability of a same response as a function of probe orientation in Experiment 3. 
tations rotated backward was also enhanced, because when the context (frame orientation) was rotated backward from the true-same at the time of judgment, the direction of the representational momentum shift actually reversed, and when the context was rotated forward from the truesame, the magnitude of forward representational momentum shift increased a greater amount than was observed in Experiment 1. Alternatively, perhaps the change in context between the third inducing stimulus (in which the frame and target were at identical orientations) and the probe (in which the frame and target may have been at different orientations) affected the magnitude and direction of representational momentum. Such a change of context, however, would be due more to changes in the relationships between elements of the display and not necessarily involve the movement of the context per se during induction.

\section{EXPERIMENT 4}

Given that representational momentum has been found in the absence of any explicit background context (e.g., Freyd \& Finke, 1984; Kelly \& Freyd, 1987), it appears that it is not the presence or absence of context per se that is important in determining whether representational momentum occurs; rather, it is the behavior of the context that is present that is critical (e.g., in Experiment 3, in which the context was in motion, the effect of surrounding context was greater than in Experiment 1, in which the context was stationary). If a background context is present, then the behavior of the context does influence the magnitude and direction of representational momentum. If no background is present, subjects may merely supply an unchanging "steady state" background by default. To examine the timing of context effects more precisely, in this experiment three different context conditions were presented to the subjects: judgment (frame was visible only during the presentation of the probe), induction (frame was visible only during the presentation of the inducing stimuli), and control (frame was never visible).

\section{Method}

Subjects. The subjects were 36 undergraduates drawn from the same pool as in Experiment 1, and none of the subjects had participated in previous experiments. Twelve subjects each participated in the judgment, induction, and control conditions.

Apparatus. The apparatus was the same as in Experiment 1.

Stimuli. The stimuli were the same as in Experiment 3, with the following exceptions: In the judgment condition, the frame was visible only during the presentation of the probe. In the induction condition, the frame was visible only during the presentation of each inducing rectangle, and each frame was drawn at the orientation of that inducing rectangle. In the control condition, the frame was never visible. Each subject received 500 trials $(2$ directions $\times 5$ probe orientations $\times 5$ frame orientations $\times 10$ replications) in a different random order.

Procedure. The procedure was the same as in Experiment 1, with the following exceptions: for the judgment condition, the frame was visible only during presentation of the probe; for the induction condition, the frame was visible only during presentation of each inducing rectangle; and for the control condition, the frame was never visible.

\section{Results and Discussion}

The choice probabilities are displayed in Figure 4. Shift estimates were calculated as in Experiment 1 and are listed in Table 1. Because it would not be meaningful to have a frame orientation factor for either the induction or control conditions, a repeated measures ANOVA was carried out only on the memory shift estimates from the judgment condition, and a $t$ test was carried out on the memory shift estimates for the induction and control conditions. The alpha level required for significance was set at .05 .

For the judgment condition, frame orientation significantly influenced shifts $\left[F(4,44)=23.84, M S_{\mathrm{e}}=.62\right]$; post hoc Newman-Keuls tests $(p<.05)$ showed all pairwise comparisons except $\left(-4^{\circ},-2^{\circ}\right)$ were significantly different. As is clearly shown in the top panel of Figure 4, when the frame is visible only during judgment, a very strong effect of frame orientation is observed such that when the frame is rotated forward from the true-same, representational momentum is forward, but when the
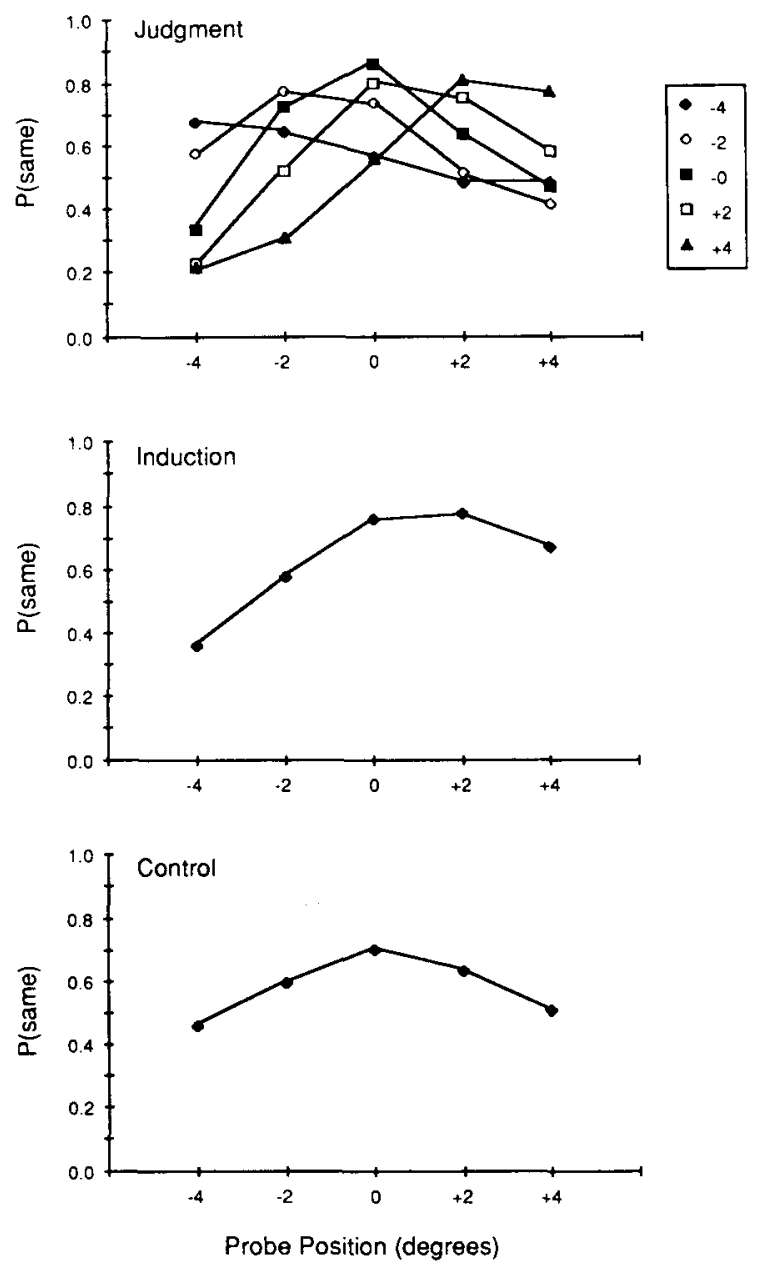

Figure 4. Probability of a same response as a function of probe orientation in Experiment 4. Data from the judgment condition are shown in the upper panel, data from the induction condition are shown in the middle panel, and data from the control condition are shown in the bottom panel. 
frame is rotated backward from the true-same, the direction of representational momentum is reversed. As is shown in the middle and bottom panels of Figure 4, when context is present during induction and moves in the same direction as the stimulus, the magnitude of forward representational momentum $(x=.56)$ is greater than when the frame is not present during either induction or judgment $(x=.08)[t(22)=2.26, p=.02]$.

Somewhat surprisingly, examination of the control group data shows little representational momentum. As previous research using the Freyd and Finke rectangle paradigm has almost always treated direction as a betweensubject variable, the very weak effects found in the current experiment may result from having treated direction as a within-subject variable. It is possible that by always treating direction as a between-subject factor, previous studies may have provided subjects with high levels of expectancy regarding the direction in which the target would travel. This expectancy might have more highly activated the representational pathways for that direction than would have happened if direction were more uncertain. Because of such relatively higher activation, a larger effect than might otherwise have been found might have been produced. This remains an area for future research, and one prediction might be that even larger effects of context might be found if direction were treated as a between-subject variable. Nevertheless, robust representational momentum was found when context was provided during either judgment or induction, and it may have been the case that the context strengthened representational momentum from the very weak levels in the control condition to more observable levels in the judgment and induction conditions.

Comparison of the judgment and control conditions shows a large effect of frame orientation; thus, the context present only at judgment can influence the pattern of representational momentum. When the frame is present during judgment, the direction of representational momentum is a function of whether the frame is rotated forward or backward from the true-same orientation of the third inducing stimulus. Comparison of the induction and control conditions shows that context present only during induction can also influence the pattern of representational momentum; specifically, when the context during induction moves in the same direction as the target, the magnitude of representational momentum is increased.

\section{EXPERIMENT 5}

If the context at the time of judgment is rotated slightly backward, forward representational momentum can be greatly diminished or even reversed. Thus, context present only during judgment is capable of reversing the direction of representational momentum. If the context is present only during induction, and that context involves motion in the same direction as the motion of the target, then the magnitude of representational momentum can be increased. It has not yet been examined whether context during induction is capable of reversing the direction of representational momentum, but previous data examining judged displacement would lead to the prediction that prior context (e.g., subjects' expectations concerning subsequent target behavior) can influence the direction of representational momentum. For example, in the Bharucha and Hubbard (1992) experiments discussed earlier, the direction and magnitude of displacement were a function of the vanishing point, such that targets vanishing just prior to or at the moment of collision were displaced backward as if subjects had already anticipated the bounce and change of direction, whereas targets that vanished just after collision (and subsequent bouncing) were displaced forward. In those data, observing the target bouncing for a number of times clearly "induced" a context in which subjects expected the target to bounce off any barriers encountered and to continue to move in a straight line until another barrier was encountered. If representational momentum is sensitive to the context present during induction, then we would predict a diminished magnitude or even a reversed direction of representational momentum when the context moves in the direction opposite to the inducing stimuli.

\section{Method}

Subjects. The subjects were 11 undergraduates drawn from the same pool as in Experiment 1, and none of the subjects had participated in the previous experiments.

Apparatus. The apparatus was the same as in Experiment 1 Stimuli. The stimuli were the same as in Experiment 3, with the following exceptions (degrees specified in counterclockwise rotation, with $0^{\circ}$ at 12:00): For clockwise trials, the frame surrounding the first presentation of the rectangle was oriented at $292^{\circ}$, the frame surrounding the second presentation of the rectangle was oriented at $309^{\circ}$, and the frame surrounding the third presentation of the rectangle was oriented at $326^{\circ}$. For counterclockwise trials, the frame surrounding the first presentation of the rectangle was oriented at $68^{\circ}$, the frame surrounding the second presentation of the rectangle was oriented at $51^{\circ}$, and the frame surrounding the third presentation of the rectangle was oriented at $34^{\circ}$. Thus, when the rectangle appeared to rotate clockwise, the frame appeared to rotate counterclockwise (and vice versa) and the frame and rectangle were aligned at an orientation that was identical to that of the third inducing stimulus. Each subject received 100 trials (2 directions $x$ 5 probe orientations $\times 10$ replications) in a different random order.

Procedure. The procedure was the same as in Experiment 1, with the following exception: the frame was visible only during the presentation of each inducing stimulus and was not visible during the presentation of the probe or during the ISIs between presentations.

\section{Results and Discussion}

The choice probabilities are displayed in Figure 5. Shifts were calculated as in Experiment 1. The average shift was $-.38^{\circ}$, and this value did not differ significantly from zero $[t(10)=-1.65, p=.13]$. The magnitude of the shifts is somewhat reduced from that seen in Experiment 3 (in which motion during induction was in the same direction as the motion of the target), and this slight decrease may reflect contributions of motion during induction. When we compare the shifts in Experiment 5 (in which context was absent during judgment but moved in 


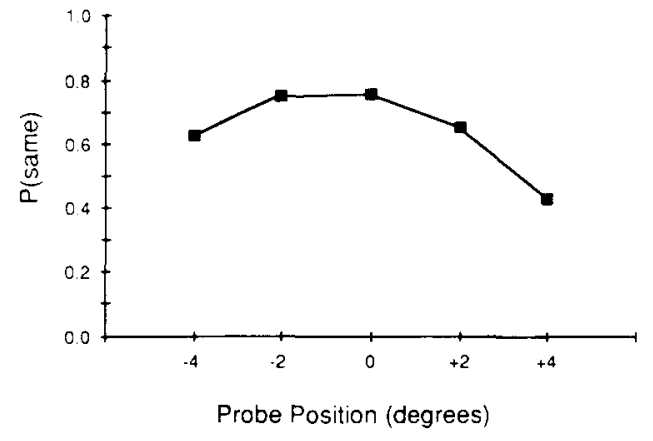

Figure 5. Probability of a same response as a function of probe orientation in Experiment 5.

the direction opposite to the target during induction) with the shifts in the induction condition in Experiment 4 (in which context was absent during judgment but moved in the same direction as the target during induction), we see that when the context moves in the direction opposite to the direction of target motion $(x=-.38)$, representational momentum of the target is significantly less than when the context moves in the same direction as the target $(x=.56)[t(21)=3.49, p=.002]$. Even so, the effects of motion of the context during induction seem relatively weak when compared with the effects of context at judgment.

Although the shifts in the current experiment were not significantly different from zero, shifts were significantly different from those obtained in the induction condition of Experiment 4, in which the context moved in the same direction as target motion. This pattern is also consistent with the very weak shift in the control condition in Experiment 4 , as a condition in which there is no context would be expected to be intermediate to a condition in which the context moves in the direction of target motion and a condition in which the context moves in the direction opposite to target motion. This is also consistent with the notions that the context during induction amplified the degree of representational momentum and that the direction of motion determined the direction of the shift. Specifically, when context during induction moved in the direction of target motion (Experiment 4), representational momentum was increased, but when context during induction moved in the direction opposite to target motion, representational momentum was not as strong and the direction of shift may have reversed.

It should be pointed out, however, that counter-rotation of the frame and target might have been predicted to produce additional induced motion of the target. Presumably this additional induced motion would have been added to the actual target motion to produce a greater extent or velocity of target motion and hence an even larger magnitude of forward representational momentum. Such effects of induced motion were not found in the overall data from the current experiment; however, it is possible that induced motion did influence perception of the target and that the subjects' judgments reflected a combination of effects from induced motion of the target and the direction of motion of the frame. Specifically, the frame may have shifted memory backward to a larger extent, but this backward shift was negated by induced motion of the target, thus resulting in a much smaller overall shift. This remains an area for additional investigation.

\section{EXPERIMENT 6}

Experiment 5 showed that if the context during induction moved in the direction opposite to the direction of motion of the target stimulus, then the direction of representational momentum could be reversed (or, at least, the magnitude of forward representational momentum decreased). Given that Experiments 1, 3, and 4 have shown that the context at judgment can also influence the direction of representational momentum, it is of interest to examine more closely the relative contributions of context during induction and context during judgment; specifically, if the context during judgment is rotated forward (and so should bias representational momentum forward), can that judgment context overcome the effects of an induction context in which the motion of the context is in the direction opposite to the motion of the target stimulus (and so should bias representational momentum backward)?

\section{Method}

Subjects. The subjects were 10 undergraduates drawn from the same pool used in previous experiments, and none of the subjects had participated in previous experiments. Data from 2 additional subjects were discarded because of the subjects' failure to follow instructions.

Apparatus. The apparatus was the same as in Experiment 1.

Stimuli. The stimuli were the same as in Experiment 5, with the following exceptions: the frame was also presented concurrently with the probe and was presented at one of five orientations relative to the third inducing stimulus: $-4^{\circ},-2^{\circ}, 0^{\circ},+2^{\circ}$, or $+4^{\circ}$. Each subject received 500 trials ( 2 directions $\times 5$ probe orientations $\times 5$ frame orientations $\times 10$ replications) in a different random order.

Procedure. The procedure was the same as in Experiment 5, with the following exception: the frame was also presented concurrently with the probe.

\section{Results and Discussion}

The choice probabilities are displayed in Figure 6. Shift estimates were calculated as in Experiment 1 and are listed in Table 1 . The shift estimates were analyzed in a 2 (direction) $\times 5$ (frame orientation) repeated measures ANOVA, and the alpha level required for significance was set at .05 . Frame orientation significantly influenced memory shift $\left[F(4,36)=10.85, M S_{\mathrm{e}}=.21\right]$, and post hoc Newman-Keuls tests revealed that all pairwise comparisons except $\left(-4^{\circ},-2^{\circ}\right),\left(-2^{\circ}, 0^{\circ}\right),\left(0^{\circ},+2^{\circ}\right)$, and $\left(+2^{\circ},+4^{\circ}\right)$ were significant. As is shown in Figure 6 , when the frame during judgment is rotated backward, the direction of representational momentum reverses, but when the frame during judgment is rotated forward, the direction of representational momentum is the same as 


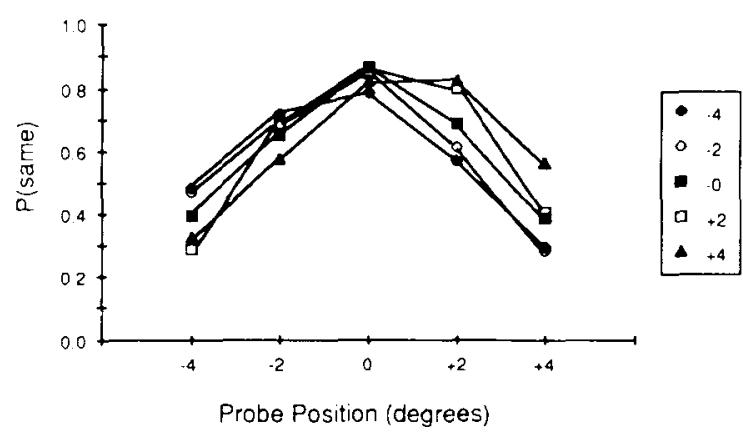

Figure 6. Probability of a same response as a function of probe orientation in Experiment 6.

the direction of motion of the target stimulus. Neither direction nor the direction $\times$ frame orientation interaction was significant $(F \mathrm{~s}<2.4, p \mathrm{~s}>.06)$.

When the shift estimates from Experiment 6 (in which context during induction moved in the direction opposite to the target) are compared with the shift estimates from the judgment condition in Experiment 4 (no context during induction) and the shift estimates from Experiment 3 (in which context during induction moved in the same direction as the target), the behavior of the context at judgment and during induction are found to influence shift magnitude as shown by a significant effect of frame orientation $\left[F(4,128)=41.88, M S_{\mathrm{e}}=.65\right]$ and a significant experiment $\times$ frame orientation interaction $[F(8,128)=$ $\left.2.83, M S_{c}=.65\right]$. As is clearly shown in Figure 7, shifts are positive when the frame at judgment is oriented beyond the true-same, but shifts are reduced in magnitude or negative when the frame at judgment is oriented behind the true-same. Also, shifts are generally positive when context is present during induction and moves in

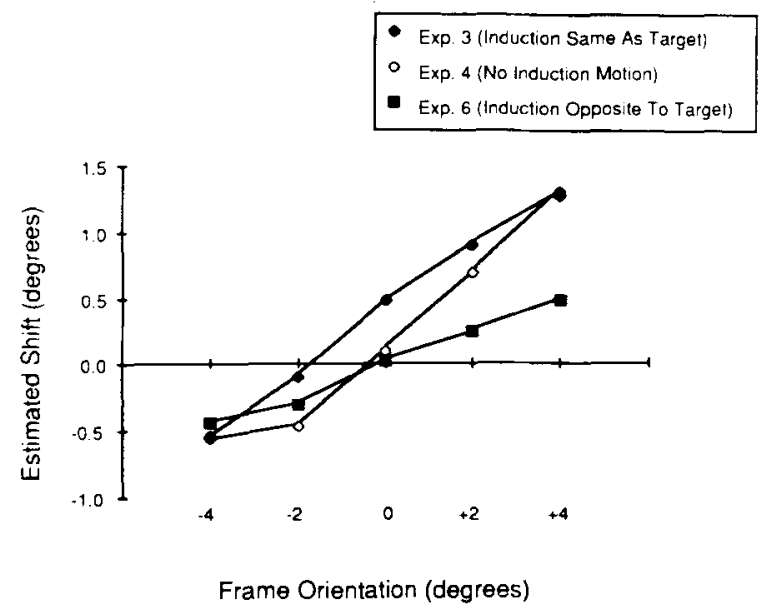

Figure 7. Estimated memory shift as a function of probe orientation. The data are from Experiment 3 (in which context during induction moved in the same direction as the target motion), the judgment condition in Experiment 4 (no context during induction), and Experiment 6 (in which context during induction moved in the direction opposite to the motion of the target). the same direction as the target or when no context is present, but shifts are generally decreased when context is present during induction and moves in the opposite direction to the target. In addition, the direction $\times$ frame orientation interaction was significant $[F(2,32)=3.93$, $M S_{\mathrm{e}}=.27$ ], such that effects of frame orientations were slightly larger on clockwise motion than on counterclockwise motion. No other factors were significant (all $F$ s < 1.7 , all $p s>.21$ ).

The data clearly show that the context in which an object is embedded can influence the pattern of representational momentum for that object. It can be clearly seen in Figure 7 that the orientation of the context at judgment has a large influence on the magnitude and direction of representational momentum regardless of the motion of the inducing context; specifically, if the context suggests an orientation slightly beyond the true-same orientation, forward representational momentum is increased, and if the context suggests an orientation slightly behind the true-same, representational momentum is decreased or reversed. The data from Experiments 3, 4, and 6 are not identical, however, suggesting that motion of the context during induction may have some attenuating effect on representational momentum, especially when that motion is in the direction opposite to the target motion.

\section{GENERAL DISCUSSION}

The context present during induction and the context present during judgment were both shown to influence the direction and magnitude of representational momentum. When the context at judgment is such as to suggest an orientation slightly beyond the true-same orientation, forward representational momentum is increased; when the context at judgment is such as to suggest an orientation slightly behind the true-same orientation, forward representational momentum is decreased or the direction of representational momentum may be reversed. One interesting point is that the reversal of forward representational momentum when the context during judgment is rotated backward occurred even in the absence of physical motion or induced motion in the opposite direction, and in the absence (presumably) of any belief or expectation of motion in the opposite direction. Therefore, this reversal may demonstrate a change in the direction of representational momentum that is not necessarily dependent upon expectations concerning future target behavior and thus may be evidence of an impenetrable aspect of representational momentum (see Finke \& Freyd, 1989). When the context during induction moves in the same direction as the target stimulus, the magnitude of forward representational momentum for the target stimulus may be increased, but when the context during induction moves in the direction opposite to the target stimulus, the magnitude of forward representational momentum for the target stimulus may be decreased or the direction of representational momentum may be reversed.

It is possible that changes in the pattern of representational momentum may reflect perceived changes in target 
identity or complexity rather than changes in the context surrounding the target. The subjects may have experienced a change in the context between the third inducing stimulus and the probe and interpreted this as a change in target identity. For example, in Experiment 2, a frame was visible during the presentation of the third inducing stimulus, but a frame was not visible during the presentation of the probe; in Experiments 3, 5, and 6, the orientations of the frame and the target were identical during the presentation of the third inducing stimulus, but the orientations of the frame and target were not necessarily identical during the presentation of the probe. Similarly, the rectangle and frame may have been perceived not as a figural target and a background context, but rather as a single larger or more complex figure undergoing rotation. Such a change in identity, rather than motion during induction or orientation during judgment per se, might have produced the observed shifts. However, to the extent that the identity or complexity had been perceived to have changed with any change in context, we would have expected general decreases in representational momentum (viz., Kelly \& Freyd, 1987), and the larger changes in identity (i.e., frame at $+4^{\circ}$ ) should have resulted in less representational momentum than the smaller changes in identity (i.e., frame at $+2^{\circ}$ ). Given that representational momentum was related more to the direction of frame orientation relative to the true-same orientation and that the magnitude of representational momentum increased with increasing distance between the frame orientation and the true-same orientation, this alternative is not supported.

One possible explanation for the effects of the context across the experiments builds on the notions of Bharucha and Hubbard (1992), Faust (1990), and Grossberg and Rudd (1989). Specifically, different areas of space are represented by different nodes within a neural network. As a target moves across space, it traces a path of activation through the network. Once a node is activated, activation spreads outward from that node. As a target moves, it spreads additional activation along the path of anticipated motion while residual activation behind the target fades. For example, consider a subject who observes a target moving from $A$ to $B$ to $C$. As the target moves, it activates neural network nodes $A^{\prime}, B^{\prime}$, and $C^{\prime}$ at times $T_{1}, T_{2}$, and $T_{3}$. At $T_{1}, A^{\prime}$ would be active, and activation would spread to $B^{\prime}$ and $C^{\prime}$. At $T_{2}$, the target has moved beyond $A^{\prime}$, and now $B^{\prime}$ is activated. At $T_{2}$, little activation would be spreading to $A^{\prime}$, while additional activation would still be spreading to $C^{\prime}$. At $T_{2}$, then, $C^{\prime}$ would possess more activation than $A^{\prime}$. Activation in front of the moving target (i.e., in the direction of anticipated target motion), therefore, would be higher than activation behind the target. The direction and magnitude of representational momentum would be determined by which network pathway possessed the highest activation. In the case in which only a single target stimulus is presented, the pathway possessing the highest activation would also correspond to the anticipated direction of target motion.
Other moving elements of the display (e.g., the frame) would also activate portions of the network. If the context moves in the same direction as the target, there would be even greater overall activation in the nodes representing the space in front of the target, and hence representational momentum would be increased (Experiment 3 ). If the context moves in the direction opposite to the motion of the target, the context may provide additional activation behind the target, and representational momentum would be diminished or reversed (Experiment 5). If the context at judgment is shifted forward relative to truesame, there would be more activation beyond the truesame, and hence representational momentum would be increased (Experiments 1-6); if the context at judgment is shifted backward, there would be more activation behind the true-same and less difference in activation between the area in front of the target and the area behind the target, thus resulting in decreased representational momentum (Experiments 1-6). The apparently stronger effect of context during judgment than during induction (Experiments 1-6) can be accounted for by positing a decay function such that activation values decay toward zero after the context is removed (or peak shortly after removal of the context and then decay; cf. Freyd \& Johnson, 1987); thus, by the time of judgment, the context during judgment would still have relatively high levels of activation (as the context during judgment is still visible), but the context during induction would have disappeared and its activation decayed somewhat, resulting in the induction context contributing less than the judgment context to the ultimate remembered location or orientation.

This type of framework can also account for other findings in the representational momentum literature (see also discussion in Hubbard, 1992c). For example, Verfaillie and d'Ydewalle (1991) showed that a standard representational momentum shift was observed when a target exhibited aperiodic motion or was far from the boundary of a periodic sequence, but that representational momentum decreased to near zero when a target was near the boundary of a periodic motion. Representational momentum in the former conditions can be accounted for with the mechanisms already described, whereas the decrease in representational momentum in the latter condition can be accounted for if we allow subjects' anticipations of future target motion to activate nodes in the network. Anticipation of periodic motion (and impending reversal of target motion) could spread increasing activation to locations behind the target (i.e., in the direction of anticipated future motion) as the target approached the boundary of the periodic motion. Activation behind the target would grow relatively stronger, and the area of highest activation (and hence the direction of representational momentum) would move from in front of the target to closer to the target's true position. If activation behind the target is very strong, and perhaps coupled with an inhibition of nodes representing locations beyond the boundary of the periodic motion, then the area of highest activation would 
move from in front of the target to behind the target. Similarly, the displacement patterns in the Bharucha and Hubbard (1992) data can be accounted for with the same mechanism: as the target approached the wall of the box and subjects anticipated a bounce, activation would spread to the nodes corresponding to the anticipated future path of the target. At the moment of collision with the wall of the box, activation would be highest in the direction of the anticipated bounce and locations beyond the walls of the box would be strongly inhibited, and thus the judgments of the target's location would be displaced in the direction consistent with the target having "bounced."

The neural network framework also leads to predictions of other possible findings. For example, not all other nodes will be equally distant from the nodes that represent the target. Nodes that are farther away may have weaker connections to the target nodes or may take longer to demonstrate an effect on the target nodes. Thus, we could predict that context that is farther away from the target may have a lesser effect than context that is closer to the target. More specifically, a smaller frame that enclosed a target might have more overall effect on representational momentum of the target than a large frame that enclosed the target. Such an effect may have already been foreshadowed in the data for the upright condition in Experiment 1 , as the relatively close upright frame led to a relatively large shift.

One unresolved issue concerns whether the effects of context are due to memorial or perceptual processes. When the frame is present during judgment, it may indeed bias representational momentum; because the frame is available to the subject, however, its effects could be considered perceptual. Similarly, frame orientation at judgment might influence perception of the probe rather than memory for the inducing stimulus. Given that Freyd and Johnson (1987) have shown that the magnitude of representational momentum (in the absence of explicit context) is a function of retention interval, however, it can be argued that representational momentum per se must be memorial. If similar effects of retention interval can be found for the effects of context, then we might be more justified in considering context effects as memorial phenomena (as the effects would be postperceptual). It may be that while representational momentum per se is memorial, factors that contribute to or modify representational momentum may be either memorial (e.g., expectations based on observations of previous target behavior) or perceptual (e.g., biasing effects of landmarks); thus, representational momentum could involve both memorial and perceptual components. At this point, the data do not yet clearly distinguish between memorial and perceptual explanations, although Hubbard and Bharucha (1988; see also Hubbard, 1992d) have argued that displacements in judged location must reflect the operation of a cognitive mechanism rather than a purely perceptual mechanism and have based this argument on the finding that judgments are displaced in the direction of anticipated motion rather than in the direction of physical motion. Untangling the relative contributions of both memory and perception for representational momentum remains a task for future investigation.

Previous research has shown that context in the form of subjects' prior experience with the type of stimulus materials or with the behavior of the specific target stimuli in a given type of stimulus display can influence the direction and magnitude of representational momentum. The experiments reported here extend the role of context and demonstrate that context in the form of other, noninteractive, physical elements present in the stimulus display can also influence the direction and magnitude of representational momentum, as the background context in which a target stimulus is embedded can clearly contribute to the direction and magnitude of any memory shifts. Consideration of the individual motion of a single local target is thus insufficient to specify the pattern of representational momentum (see also Verfaillie \& d'Ydewalle, 1991); indeed, specification of several levels of context, including subjects' prior schematic knowledge about the stimuli, subjects' expectations based on observing the stimuli, and the behavior of other elements of the stimulus display, is required before the memory shift pattern can be fully understood.

\section{REFERENCES}

Bharucha, J. J., Hubbard, T. L. (1992). Anticipated collision along an oblique path: Effects on judged displacement. Manuscript submitted for publication.

FAUST, M. (1990). Representational momentum: A dual process perspective. Unpublished doctoral dissertation, University of Oregon, Eugene.

FinKE, R. A., \& FRYyd, J. J. (1985). Transformations of visual memory induced by implied motions of pattern elements. Journal of $E_{x}$ perimental Psychology: Leaming, Memory, \& Cognition, 11, 780-794.

FINKE, R. A., \& FrEYD, J. J. (1989). Mental extrapolation and cognitive penetrability: Reply to Ranney and proposals for evaluative criteria. Journal of Experimental Psychology: General, 118, 403-408.

Finke, R. A., FreYd, J. J., ShYI, G. C. W. (1986). Implied velocity and acceleration induce transformations of visual memory. Journal of Experimental Psychology: General, 115, 175-188.

FreYD, J. J., \& FinKE, R. A. (1984). Representational momentum. Journal of Experimental Psychology: Learning, Memory, \& Cognition, 10, 126-132.

FREYD, J. J., \& FINKE, R. A. (1985). A velocity effect for representational momentum. Bulletin of the Psychonomic Society, 23, 443-446.

FreYd, J. J., \& Johnson, J. Q. (1987). Probing the time course of representational momentum. Journal of Experimental Psychology: Learning, Memory, \& Cognition, 13, 259-268.

Freyd, J. J., Kelly, M. H., DeKay, M. L. (1990). Representational momentum in memory for pitch. Joumal of Experimental Psychology: Learning, Memory, \& Cognition, 16, 1107-1117.

Grossberg, S., \& RUdD, M. E. (1989). A neural architecture for visual motion perception; Group and element apparent motion. Neural Networks, 2, 421-450.

Hubbard, T. L. (1990). Cognitive representation of linear motion: Possible direction and gravity effects in judged displacement. Memory \& Cognition, 18, 299-309. 
HubBard, T. L. (1992a). Auditory representational momentum: Musical schemata and modularity. Manuscript submitted for publication.

HubBa RD, T. L. (1992b). Auditory representational momentum: Sur face form, velocity, and direction effects. Manuscript submitted for publication.

HubBarD, T. L. (1992c). Cognitive representation of motion: Evidence for friction and gravity analogues. Manuscript submitted for publication.

HuBBaRD, T. L. (1992d). Judged displacement: A modular process? Manuscript submitted for publication.

Hubbard, T. L., \& Bharucha, J. J. (1988). Judged displacement in apparent vertical and horizontal motion. Perception \& Psychophysics, 44, 211-221.

Kelly, M. H., FREYD, J. J. (1987). Explorations of representational momentum. Cognitive Psychology, 19, 369-401.

VerfaILLIE, K., \& D'Ydewalle, G. (1991). Representational momentum and event course anticipation in the perception of implied periodical motions. Journal of Experimental Psychology: Learning, Memory, \& Cognition, 17, 302-313.

(Manuscript received March 17, 1992; revision accepted for publication August 4, 1992.) 